\title{
The ability of lipopolysaccharide (LPS) of Pasteurella multocida B:2 to induce clinical and pathological lesions in the nervous system of buffalo calves following experimental inoculation
}

\begin{abstract}
Lipopolysaccharide (LPS) of P. multocida B:2, a causative agent of haemorrhagic septicaemia (HS) in cattle and buffaloes, is considered as the main virulence factor and contribute in the pathogenesis of the disease. Recent studies provided evidences about the involvement of the nervous system in pathogenesis of HS. However, the role of P. multocida B:2 immunogens, especially the LPS is still uncovered. Therefore, this study was designed to investigate the role of P. multocida B:2 LPS to induce pathological changes in the nervous system. Nine eight-month-old, clinically healthy buffalo calves were used and distributed into three groups. Calves of Group 1 and 2 were inoculated orally and intravenously with $10 \mathrm{ml}$ of LPS broth extract represent $1 \times 1012 \mathrm{cfu} / \mathrm{ml}$ of P. multocida B:2, respectively, while calves of Group 3 were inoculated orally with $10 \mathrm{ml}$ of phosphate buffer saline as a control. Significant differences were found in the mean scores for clinical signs, post mortem and histopathological changes especially in Group 2, which mainly affect different anatomic regions of the nervous system, mainly the brain. On the other hand, lower scores have been recorded for clinical signs, gross and histopathological changes in Group 1. These results provide for the first time strong evidence about the ability of P. multocida B:2 LPS to cross the blood brain barrier and induce pathological changes in the nervous system of the affected buffalo calves.
\end{abstract}

Keyword: Haemorrhagic septicaemia; Buffalo calves; Pasteurella multocida B:2; Lipopolysaccharide (LPS); Nervous system; Clinical; Pathological 